\title{
Patch-Based Markov Models for Event Detection in Fluorescence Bioimaging
}

\author{
Thierry Pécot ${ }^{1,2}$, Charles Kervrann ${ }^{1,2}$, Sabine Bardin ${ }^{3}$, Bruno Goud ${ }^{3}$, \\ and Jean Salamero ${ }^{3,4}$
}

\footnotetext{
${ }^{1}$ INRIA Rennes - Bretagne Atlantique, Campus de Beaulieu, F-35042 Rennes

${ }^{2}$ INRA, UR341 Mathématiques et informatique appliquées, F-78352 Jouy-en-Josas

${ }^{3}$ UMR 144 CNRS - Institut Curie, F-75248 Paris

4 "Cell and Tissue Imaging Facility" - IBISA, Institut Curie, F-75248 Paris
}

\begin{abstract}
The study of protein dynamics is essential for understanding the multi-molecular complexes at subcellular levels. Fluorescent Protein (XFP)-tagging and time-lapse fluorescence microscopy enable to observe molecular dynamics and interactions in live cells, unraveling the live states of the matter. Original image analysis methods are then required to process challenging $2 \mathrm{D}$ or $3 \mathrm{D}$ image sequences. Recently, tracking methods that estimate the whole trajectories of moving objects have been successfully developed. In this paper, we address rather the detection of meaningful events in spatio-temporal fluorescence image sequences, such as apparent stable "stocking areas" involved in membrane transport. We propose an original patch-based Markov modeling to detect spatial irregularities in fluorescence images with low false alarm rates. This approach has been developed for real image sequences of cells expressing XFP-tagged Rab proteins, known to regulate membrane trafficking.
\end{abstract}

\section{Introduction}

To preserve the structure, cohesion and functions of the organism, the eukaryotic cell exchanges information between its compartments and organelles: endosomes, Golgi apparatus, endoplasmic reticulum (ER), ..., these intracellular exchanges require physical supports or networks for communication, mainly cytoskeleton elements such as microfilaments or microtubules. Membrane interactions with microtubules are mediated by several classes of proteins, notably motor proteins of the dynein and kinesin families. In our study, the transport intermediates/vesicles corresponding to small (less than 100nm) and almost spherical elements in time-lapse fluorescence microscopy move along microtubules. Molecular motors transform chemical energy with the mediation of Adenosine Tri-Phosphate (ATP) to mechanical work and driving energy for propelling the vesicles. We focus on the traffic between two cell compartments - the Golgi apparatus and "peripheral structures" - presumably regulated by two isoforms of the Rab6 GTPase (Rab6A and Rab6A') [1. Characterization of the latter structures is still elusive so far and a matter of debate. However, they are believed to correspond to Endoplasmic Reticulum "entry points" in eukariotic cells. We name them

D. Metaxas et al. (Eds.): MICCAI 2008, Part II, LNCS 5242, pp. 95-103, 2008.

(C) Springer-Verlag Berlin Heidelberg 2008 
"end-points" since they correspond to areas where Rab6 labeled transport intermediates appear to be delivered. Observation of protein dynamics in live cells using XFP-tagging and time-lapse fluorescence microscopy is routinely used to elucidate the roles of Rab proteins in membrane transport. In particular, XFP-Rab6 proteins are either free (diffusion) in the cytosol, anchored to the moving transport intermediates, transiently attached to the Golgi membranes ("origin" region) or present near the so-called "end-points" ("destination" regions).

Image processing methods have been developed to reliably and successfully track the transport intermediates along microtubules over time [7. The most commonly-used tracking approach is based on motion correspondence [23]: the particles are detected independently in each frame in a first step, and then the trajectories are computed by connecting the detected objects over time. Sophisticated particle filtering techniques [45], graph-theory based methods [6] or minimal paths methods [7] have been also developed to improve temporal matching. In this paper, we address the problem of spatial detection of fluorescence irregularities in 2D images of temporal sequences obtained in time-lapse fluorescence microscopy. Our idea is to robustly determine the "origin" and "destination" regions (OD regions) involved in vesicular trafficking. Detection methods with low false alarm rates are then required to provide new tools for investigation in fluorescence bioimaging. Therefore, we propose here an original patch-based Markov Random Field (MRF) modeling. It turns out that this method allows to detect reliably the OD points unlike previous methods. The remainder of the paper is organized as follows: in section 2, we describe the patch-based MRF model. In section 3. we propose a statistical method to automatically threshold the potential map. In section 4 , we present experiments on real image sequences.

\section{Patch-Based Markov Models for Image Representation}

In our study, membrane transport correspond to vesicles moving from "origin" to "destination" regions. In the background, fluorescence diffusion represents the largest component and moves slowly when compared to the vesicle traffic component. However, apparent stable "stocking" areas corresponding to areas with accumulated fluorescence should be detected. Our idea is then to detect pixels with meaningful fluorescence for several frames. A temporally cumulated detection map will be derived further to detect the OD regions / "stocking" areas.

A first approach to measure the fluorescence regularity in each image is to compute the gradient amplitude image. Since it is established that fluorescence is proportional to the number of proteins, the higher the gradient amplitude is, the bigger the concentration of small particles is. Nevertheless, the gradient is also very sensitive to low signal-to-noise ratios and undesirable artifacts are also detected (see Fig. 1b)). New statistical models are then required to improve irregularity detection in each image.

In our approach, we adopt a MRF framework [8] to capture image regularity. In contrast to the usual pixel-wise MRF models, a recent line of work consists in modeling non-local interactions from image patches; in 91011, the redundancy 
property and patch-based representation can be exploited to detect unusual spatial patterns seen in the scene. In our study, this property holds true and we also propose an original patch-based Gibbs/MRF modeling to represent the more regular image components. We detect further the locations where redundancy is low, that is protein concentrations against a nearly uniform background ideally. In the presence of noise, patch-wise Markov models produce potential maps which are more regular than those obtained with pixel-wise Markov models. As we shall see in our experiments, considering non-local interactions enables to better describe the image contents.

More formally, consider a gray-scale image $u=(u(x))_{x \in \Omega}$ defined over a bounded domain $\Omega \subset \mathcal{R}^{2}$ and defined at pixel $x \in \Omega$ as $u(x)=u_{0}(x)+\varepsilon(x)$, where $u_{0}$ is the true image and the errors $\varepsilon(x)$ are assumed to be iid (independent identically distributed) Gaussian zero-mean random variables with unknown variance $\sigma^{2}$. Note that the assumption of Gaussian noise holds true in fluorescence microscopy provided the number of photons collected at each pixel is large enough. In order to robustly detect redundancy in $u$, we focus on image patches as non-local image features able to capture spatial regularities. Our idea is to perform pairwise comparisons of $n$-dimensional patches $\underline{u}(x)$ within a fixed-size semi-local neighborhood. For the sake of simplicity, a vectorized image patch $\underline{u}(x)$ is defined as the $\sqrt{n} \times \sqrt{n}$ square neighborhood of point $x$ and the pixels are ordered lexicographically. In [9], it has been confirmed that the $l_{2}$ distance $\|\underline{u}(x)-\underline{u}(y)\|^{2}$ is able to express the amount of similarity between image patches $\underline{u}(x)$ and $\underline{u}(y)$. Intuitively, if the distance is large enough, we can conclude that the patches centered at pixels $x$ and $y$ belong to different spatial contexts. Besides, in homogeneous regions, the noise being assumed to be Gaussian, the score $z(x, y) \triangleq \frac{\|\underline{u}(x)-\underline{u}(y)\|^{2}}{2 \sigma^{2}}$ follows a central chi-squared distribution with $n$ degrees of freedom, i.e. $z(x, y) \sim \chi_{n}^{2}$.

The key idea here is to combine the MRF framework with patch-based representation. Instead of defining the underlying potential function by hand or training [10], we exploit patch-based score statistics since $z(x, y)$ is expected to follow a central chi-squared distribution. Let $G=(V, E)$ be a graph where $V$ denotes the nodes and $E$ the edges connecting the nodes. Moreover, a neighborhood system connecting all the nodes in the square window (larger than patches) and centered at pixel $x$, is defined. The Hammersley-Clifford theorem establishes that the pdf of the proposed graphical model is a Gibbs distribution of the form $p(u) \propto \exp -\sum_{<x, y\rangle} \phi(x, y)$ where $\langle x, y\rangle$ denotes the set of cliques in the neighborhood and $\phi(x, y)$ is the assumed homogeneous local interaction potential function. We arbitrarily choose $\phi(x, y)=\log (p(z(x, y)))$ and write the pdf of $u$ as

$$
p(u) \propto \exp -\left[\sum_{<x, y>}(n-2) \log (\|\underline{u}(x)-\underline{u}(y)\|)-\frac{\|\underline{u}(x)-\underline{u}(y)\|^{2}}{4 \sigma^{2}}\right] .
$$

In what follows, all the pairwise comparisons between neighboring patches wrt vertical and horizontal directions will be considered. This Gibbs model is parametrized by only one parameter, i.e. the noise variance $\sigma^{2}$, and will directly 


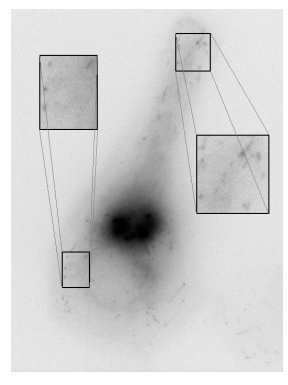

a)

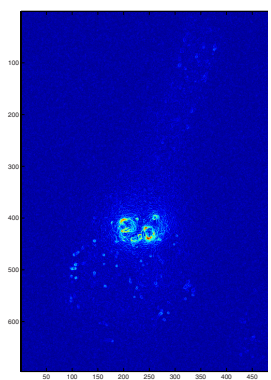

b)

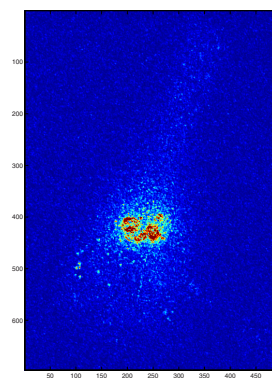

c)

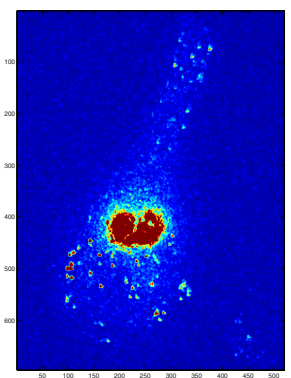

d)

Fig. 1. a) Processing of a typical image (and zoomed-in views) extracted from a timelapse microscopy image sequence where high fluorescence levels correspond to dark values (a gamma correction is applied for better visualization); b) gradient amplitude image; c) potential map using pixel-wise MRF modeling; d) potential map using patchwise MRF modeling. In figures b)-d), blue regions correspond to low potential values and red regions to high potential values.

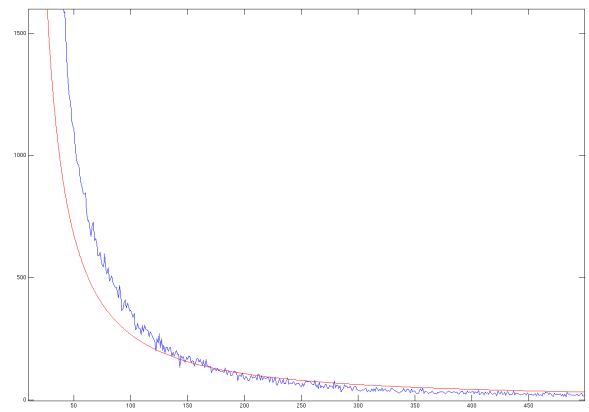

a)

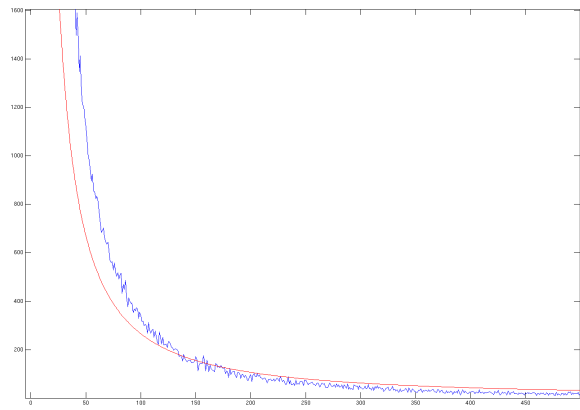

b)

Fig. 2. Approximation of the observed pdf (blue) of $\Phi$ by a mixture of two distributions (red) for images \#10 (a)) and \#50 (b)) in the sequence shown in Fig. 1]

estimated by Maximum Likelihood (ML) from data points belonging to the background. As explained in [10], the normalization term involved in the Gibbs model (1) is intractable but is not required for our purpose, as we shall see later. The proposed Gibbs distribution also includes spatial correlation in the modeling since the neighboring patches overlap in the neighborhood.

For illustration, Fig. 1 shows respectively the gradient amplitude image (Fig. 1b)), the potential map (Fig. 15)) using the homogeneous pixel-wise Gibbs model defined as $p(u) \propto \exp -\beta \sum_{<x, y>}(u(x)-u(y))^{2}$, with $\beta=\left(2 \sigma^{2}\right)^{-1}$, and the potential map using the patch-wise Gibbs model (Fig. 11)) when applied to an input fluorescence image shown in Fig. 17) and extracted from a time-lapse microscopy image sequence composed of 120 images. The gradient amplitude image shows high values in areas corresponding to the Golgi region (image center) 
and in smaller areas corresponding to potential "end-points". In Fig. 11c-d), a potential value $\Phi(x) \propto-\log p(\underline{u}(x) \mid \underline{u}(y), y \in \Delta(x))$ is computed at pixel $x$ from local interactions specified for both the pixel-wise and patch-wise MRF models as

$$
\Phi(x)=\sum_{y \in \Delta(x)}(n-2) \log (\|\underline{u}(x)-\underline{u}(y)\|)-\frac{\|\underline{u}(x)-\underline{u}(y)\|^{2}}{4 \sigma^{2}},
$$

where $\Delta(x)$ is the neighborhood centered at pixel $x$. The patch-wise MRF model enhances the presence of meaningful areas which could be labeled as "end-points" and the related potential map is more regular and noiseless when compared to the potential map obtained with the pixel-wise MRF model.

\section{Irregularity Detection and False Alarm Rate}

Given the noise variance $\sigma^{2}$, the patch-wise Gibbs model can be then used to assess the regularity of an input image. Based on semi-local interactions (2), a potential map $\Phi=(\Phi(x))_{x \in \Omega}$ is computed as explained above. As expected, in the presence of irregularities the potential is high, which means that the proposed model cannot wholly capture all the spatial image features. To detect irregularities, we then propose to automatically threshold the potential map. Since the image is relatively homogeneous, only a few areas correspond to high values. We want to test the hypothesis for each pixel $x$ that the potential $\Phi(x)$ is meaningful (hypothesis $H_{0}$ ) or not (hypothesis $H_{1}$ ). A very convenient way to define this notion consists in assuming that the potential $\Phi(x)$ values are independent and distributed according to the following mixture distribution:

$$
f(\Phi(x))=\delta(\Phi(x)<\epsilon) f_{0}(\Phi(x))+(1-\delta(\Phi(x)<\epsilon)) f_{1}(\Phi(x)),
$$

where $\delta(\cdot)$ denotes the Kronecker, $f_{0}$ denotes the pdf of the potential under $H_{0}$ and $f_{1}$ is the pdf of the potential under $H_{1}$. In the mixture model, $f_{0}$ is a Dirac function centered at " 0 " $\left(\epsilon=0_{+}\right.$subject to $\left.\epsilon>0\right)$ and the tail $f_{1}$ of the pdf is approximated by a Pareto distribution of the form $f_{1}(\Phi(x))=$ $\frac{k \epsilon^{k}}{(\Phi(x))^{k+1}}, \forall \Phi(x) \geq \epsilon$, with parameters $k$ and $\epsilon>0$. A Maximum Likelihood estimate for $k$ can be easily derived, i.e. $\hat{k}=N\left(\sum_{x \in \Omega: \Phi(x) \geq \epsilon} \log (\Phi(x))-\log (\epsilon)\right)^{-1}$ where $N=\#\{x \in \Omega: \Phi(x) \geq \epsilon\}$. The Pareto distribution is recommended to describe the tails of pdfs and performs well as shown in Fig. 2, Accordingly, the probability that $\Phi(x)$ is larger than a threshold $\tau \geq \epsilon$ is $\mathcal{P}\{\Phi(x) \geq \tau\}=(\tau / \epsilon)^{-k}$. Therefore, for a given false alarm probability $\mathcal{P}_{F A}:=\mathcal{P}\{\Phi(x) \geq \tau\}$ selected by the user (and assumed to be constant in the whole image), one can compute the corresponding threshold as $\tau=\exp \left(\log (\epsilon)-\frac{\log \left(\mathcal{P}_{F A}\right)}{k}\right)$.

In practice, we compute first the ML estimate $\hat{k}$ and, for a given false alarm probability $\mathcal{P}_{F A}$, we derive the thresholds $\tau$ which are different for each image in the sequence. We observed experimentally that the appropriate false alarm probability $\mathcal{P}_{F A}$ is the same for image sequences acquired in the same conditions. 


\section{Experimental Results}

First, we propose two typical experiments to illustrate the ability of our method to detect $\mathrm{OD}$ regions from cumulated detections over time. High values correspond to repeated detections in the same area. The image sequences correspond to $3 \mathrm{D}+\mathrm{T}$ time-lapse fluorescence wide-field microscopy. They are converted into $2 \mathrm{D}+\mathrm{T}$ sequences by averaging along the $z$ axis. The images are coded in 2 bytes and the voxel size is $160 \times 160 \times 300 \mathrm{~nm}^{3}$. The frame rate is equal to 1 frame/second. In all the experiments, the neighborhood size is $(p+2) \times(p+2)$ if we consider $p \times p$ patches.

In the first experiment (Fig. 3), we compare the cumulated detection maps obtained using the patch-wise MRF model (see (10) with different patch sizes.

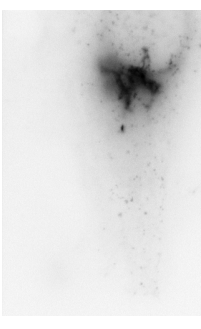

a)

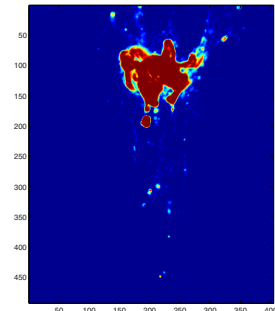

b)

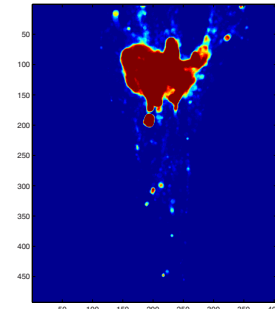

c)

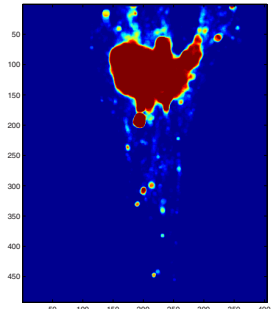

d)

Fig. 3. a) Image extracted from a second time-lapse microscopy image sequence where high fluorescence levels correspond to dark values; b)-d) cumulated detection maps using the patch-wise MRF modeling with $3 \times 3$ patches and $\left.\mathcal{P}_{F A}=0.15 \mathrm{~b}\right), 5 \times 5$ patches and $\mathcal{P}_{F A}=0.18 \mathrm{c}$ ), and $7 \times 7$ patches and $\mathcal{P}_{F A}=0.22 \mathrm{~d}$ ) (blue regions correspond to a small number of detections and red regions to a large number of detections).

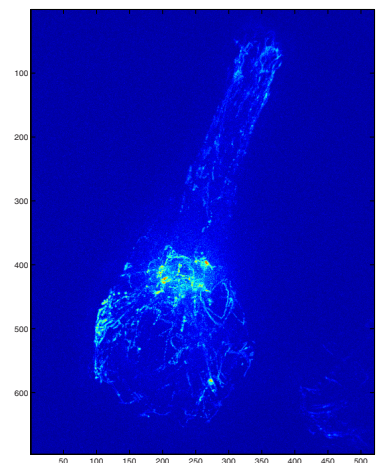

a)

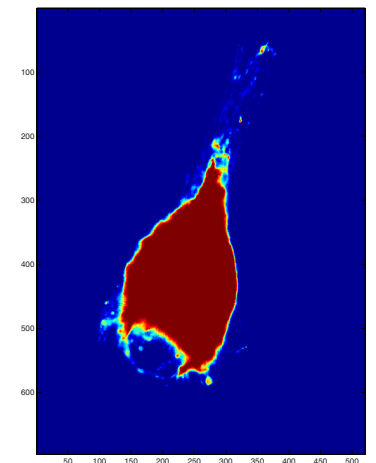

b)

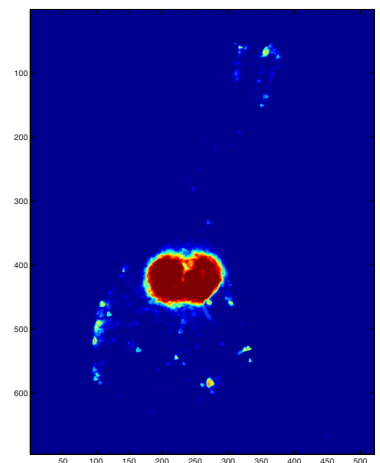

c)

Fig. 4. Processing of the image sequence shown in Fig. 1 a) MIP map; b) cumulated map corresponding to the thresholded images of the sequence; c) cumulated detection map with patch-wise MRF modeling $\left(3 \times 3\right.$ patches, $\left.\mathcal{P}_{F A}=0.15\right)$ (blue regions correspond to a small number of detections and red regions to a large number of detections). 


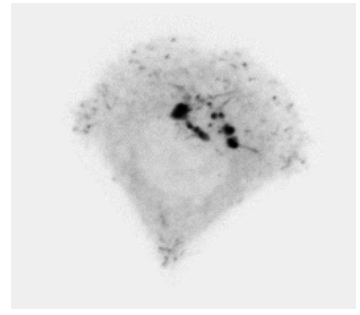

a)

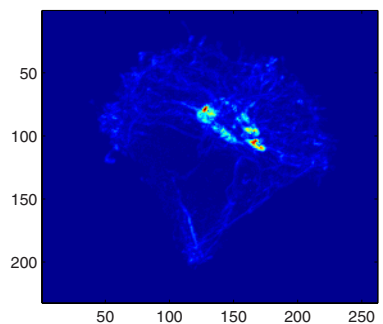

b)

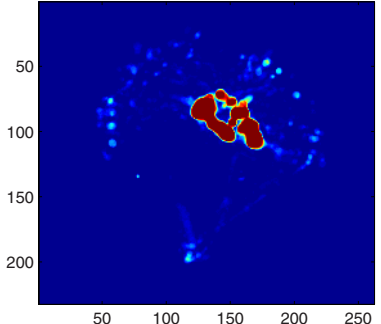

c)

Fig. 5. a) Image extracted from a spinning disk confocal microscopy image sequence where high fluorescence levels correspond to dark values; b) MIP map; c) cumulated detection map using the patch-wise MRF modeling (blue regions correspond to a small number of detections and red regions to a large number of detections)

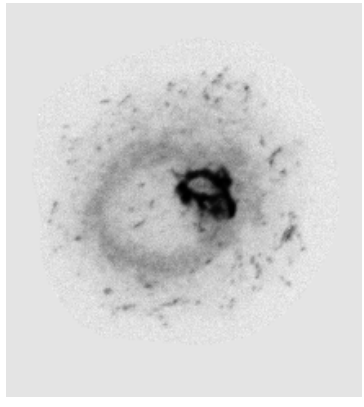

a)

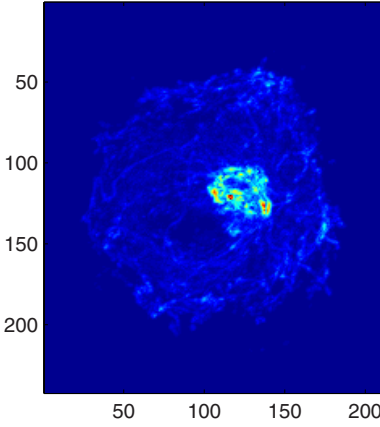

b)

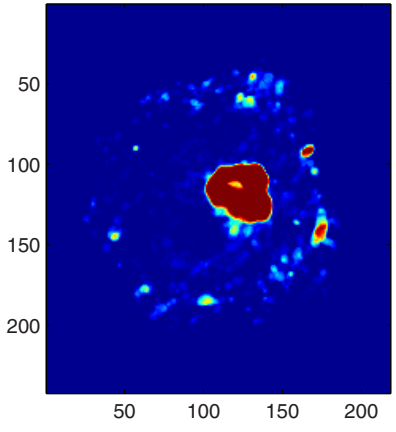

c)

Fig. 6. a) Image extracted from a spinning disk confocal microscopy image sequence where high fluorescence levels correspond to dark values; b) MIP map; c) cumulated detection map using the patch-wise MRF modeling (blue regions correspond to a small number of detections and red regions to a large number of detections)

In this experiment, it turns out that $5 \times 5$ and $7 \times 7$ patches tend to oversmooth and blur the cumulated detection map. Clearly, the "end-points" corresponding to small areas with high values cannot be individually segmented. The expected OD regions are satisfyingly extracted by using the patch-wise MRF model and $3 \times 3$ patches.

In the second experiment (Fig. 4), we compare the Maximum Intensity Projection (MIP) map, the cumulated map of the thresholded images of the sequence where each frame is thresholded independently, and the cumulated detection map obtained using the patch-wise MRF approach. The MIP map (in the direction of time axis) is a commonly-used tool to give an insight of both the traffic support and "stocking areas". At each pixel $x \in \Omega$, we define $\operatorname{MIP}(x)=$ $\max _{t \in\{1, \ldots, T\}} u_{t}(x)$, where $u_{t}(x)$ is the intensity observed at pixel $x$ and time $t$ in the image $u_{t}$ and $T$ is the number of images in the sequence. In Fig. 4a), 
the Golgi apparatus (central bright region) and several potential "end-points" (bottom left hand-side) are relatively well contrasted wrt the background. Nevertheless, several frequently used paths are also contrasted and detecting reliably the meaningful "stocking areas" is not an easy task. The thresholded frames are disturbed by the high level of fluorescence observed in the Golgi region. This implies that either the major part of the cell is detected or only the Golgi is. Finally, the patch-wise MRF model is more relevant to detect additional "endpoints" in the expected areas (see Fig. 4 4 )). This map is also more regular and the contours of "stocking areas" are better delineated.

Finally, we propose two other original experiments corresponding to $3 \mathrm{D}+\mathrm{T}$ spinning disk confocal microscopy. In these experiments, the cell shapes are constrained to have a predefined pattern (crossbow in the first sequence, cercle in the second one). This compels the cytoskeleton to have a specific spatial topology, and consequently influence the locations of the suspected "end-points".

In the first experiment (Fig. 5), the main detected regions are the Golgi and three other areas located at the left, the right and the bottom of the cell $\left(\mathcal{P}_{F A}=\right.$ 0.2 ). Additional OD regions are detected at the superior periphery of the cell. Hence, the detected areas correspond to specific points of the constrained cell shape. On the contrary, in the second experiment (Fig. 6), the detected areas seem to be uniformly distributed at the cell periphery. These two experiments tend to confirm that the cell shape influences the locations of the "end-points".

\section{Conclusion}

In this paper, we have proposed a general probabilistic and patch-based framework for irregularity detection in time-lapse fluorescence microscopy imaging. New methods are needed to detect "stocking" areas and OD regions in traffic image sequence. The cumulated detection maps enable to extract more reliably the regions of interest. In practice, this method only requires the setting of the false alarm probability. For future work, we also plan to valid this method for a large number of image sequences and to investigate this methodology for motion detection in time-lapse fluorescence microscopy.

\section{References}

1. Echard, A., Opdam, F., de Leeuw, H., Jollivet, F., Savelkoul, P., Hendriks, W., Voorberg, J., Goud, B., Fransen, J.: Alternative splicing of the human rab6a gene generates two close but functionally different isoforms. Molecular biology of the cell 11, 3819-3833 (2000)

2. Anderson, C., Georgiou, G., Morrison, I., Stevenson, G., Cherry, R.: Tracking of cell surface receptors by fluorescence digital imaging microscopy using a chargedcoupled device camera. low-density lipoprotein and influenza virus receptor mobility at 4 degrees c. Journal of Cell Science 101, 415-425 (1992)

3. Sbalzarini, I., Koumoutsakos, P.: Feature point tracking and trajectory analysis for video imaging in cell biology. Journal of Structural Biology 151, 182-195 (2005) 
4. Smal, I., Draegestein, K., Galjart, N., Niessen, W., Meijering, E.: Rao-blackwellized marginal particle filtering for multiple object tracking in molecular bioimaging. In: IPMI, pp. 110-121 (2007)

5. Genovesio, A., Liedl, T., Emiliani, V., Parak, W., Coppey-Moisan, M., OlivoMarin, J.C.: Multiple particle tracking in 3D+t microscopy: Method and application to the tracking of endocytosed quantum dots. IEEE Trans. on IP 15(5), 1062-1070 (2006)

6. Thomann, D., Dorn, J., Sorger, P., Danuser, G.: Automatic fluorescent tag localization ii: improvement in super-resolution by relative tracking. Journal of Microscopy 211(3), 230-248 (2003)

7. Racine, V., Salamero, J., Fraisier, V., Trubuil, A., Sibarita, J.-B.: Visualization and quantification of vesicle trafficking on a three-dimensional cytoskeleton network in living cells. Journal of Microscopy 225, 213-227 (2007)

8. Besag, J.: Spatial interaction and the statistical analysis of lattice systems. Journal Royal Statistical Society 36, 192-236 (1974)

9. Buades, A., Coll, B., Morel, J.: A review of image denoising algorithms, with a new one. Multiscale Modeling and Simulation 4(2), 490-530 (2005)

10. Roth, S., Black, M.J.: Fields of experts: A framework for learning image priors. In: Proc. of IEEE CVPR 2005, San Diego, USA, vol. 2, pp. 860-867 (June 2005)

11. Awate, S.P., Whitaker, R.T.: Unsupervised, information-theoretic, adaptive image filtering for image restoration. IEEE Trans. Pattern Anal. Mach. Intell. 28(3), 364$376(2006)$ 